\title{
Parallel Transjugular Intrahepatic Portosystemic Shunt (TIPS) Creation in a Patient with a Preexisting Unsalvageable Occluded TIPS and Refractory Variceal Bleeding
}

Krantikumar Rathod ${ }^{1,2}$ Bhavesh Popat ${ }^{2}$ Pankaj Barai ${ }^{1}$ Deepak Amrapurkar ${ }^{3}$

Address for correspondence Krantikumar Rathod, MD, Department of Vascular and Interventional Radiology, Bombay Hospital and Medical Research Center, Marine Lines, Mumbai, India (e-mail: krantikumarrathod@gmail.com).

\begin{abstract}
Keywords

- parallel TIPS

- TIPS dysfunction

- variceal bleeding

Transjugular intrahepatic portosystemic shun (TIPS) dysfunction is usually secondary to acute thrombosis within the stent, pseudointimal hyperplasia in the TIPS parenchymal tract, or intimal hyperplasia in the outflow hepatic vein. Several methods have been used in treating TIPS dysfunction, including balloon angioplasty, stent insertion, and the creation of a parallel TIPS. We report a case of refractory variceal bleeding in which a parallel TIPS was successfully created after failure to recanalize the preexisting occluded TIPS.
\end{abstract}

\section{Introduction}

Cirrhosis of the liver is associated with complications of portal hypertension, such as gastrointestinal bleeding and refractory ascites. The creation of a transjugular intrahepatic portosystemic shunt (TIPS) is now considered an effective therapy in managing such complications. ${ }^{1}$ TIPS dysfunction is secondary to acute stent thrombosis, pseudointimal hyperplasia, or intimal hyperplasia in the outflow hepatic vein. ${ }^{2-4}$ A nonfunctioning TIPS is treated either by balloon angioplasty, stent insertion, or creating a parallel shunt (PS). PS is the last therapeutic option when the initial shunt cannot be accessed by a guidewire or percutaneous needle. We report a case of a patient who presented with refractory variceal bleeding 5 years after chronic TIPS occlusion. The patient was successfully treated by creating a parallel TIPS after a failure to recanalize the preexisting TIPS.

\section{Case Report}

A 72-year-old man presented to our hospital with massive hematemesis. He was known to have hepatic cirrhosis for over
11 years. He had undergone TIPS creation 9 years prior to the presentation due to recurrent variceal bleeding despite endoscopic therapy. Five years prior to the presentation, he had a history of massive hematemesis. Endoscopy and duplex ultrasound at that time revealed grade 3 esophageal varices and an occluded TIPS, respectively. The measured hepatic venous pressure gradient was $23 \mathrm{~mm} \mathrm{Hg}$ and TIPS revision was unsuccessful. He was managed conservatively with endoscopic and medical therapy. On examination, the patient had splenomegaly and ascites. Laboratory values included hemoglobin of $6.7 \mathrm{~g} / \mathrm{dL}$, white cell count of $8,000 /$ cubic $\mathrm{mL}$, and platelets of $20,000 /$ cubic $\mathrm{mL}$. The total bilirubin was $3.2 \mathrm{mg} / \mathrm{dL}$, creatinine $1.6 \mathrm{mg} / \mathrm{dL}$, and international normalized ratio 1.4. The measured Model of End-Stage Liver Disease score at the time of presentation was 19.

Due to continued variceal bleeding, the patient underwent TIPS recanalization procedure through a transjugular access. This procedure was unsuccessful due to the chronicity of occluded TIPS. Percutaneous recanalization of the shunt was considered risky due to the presence of severe thrombocytopenia and ascites. A decision was made to create a parallel
DOI https://doi.org/ 10.1055/s-0036-1597653. ISSN $0000-0000$.
Copyright (c) 2017 by Indian Society of Vascular and Interventional Radiology
License terms

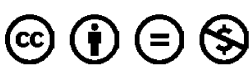



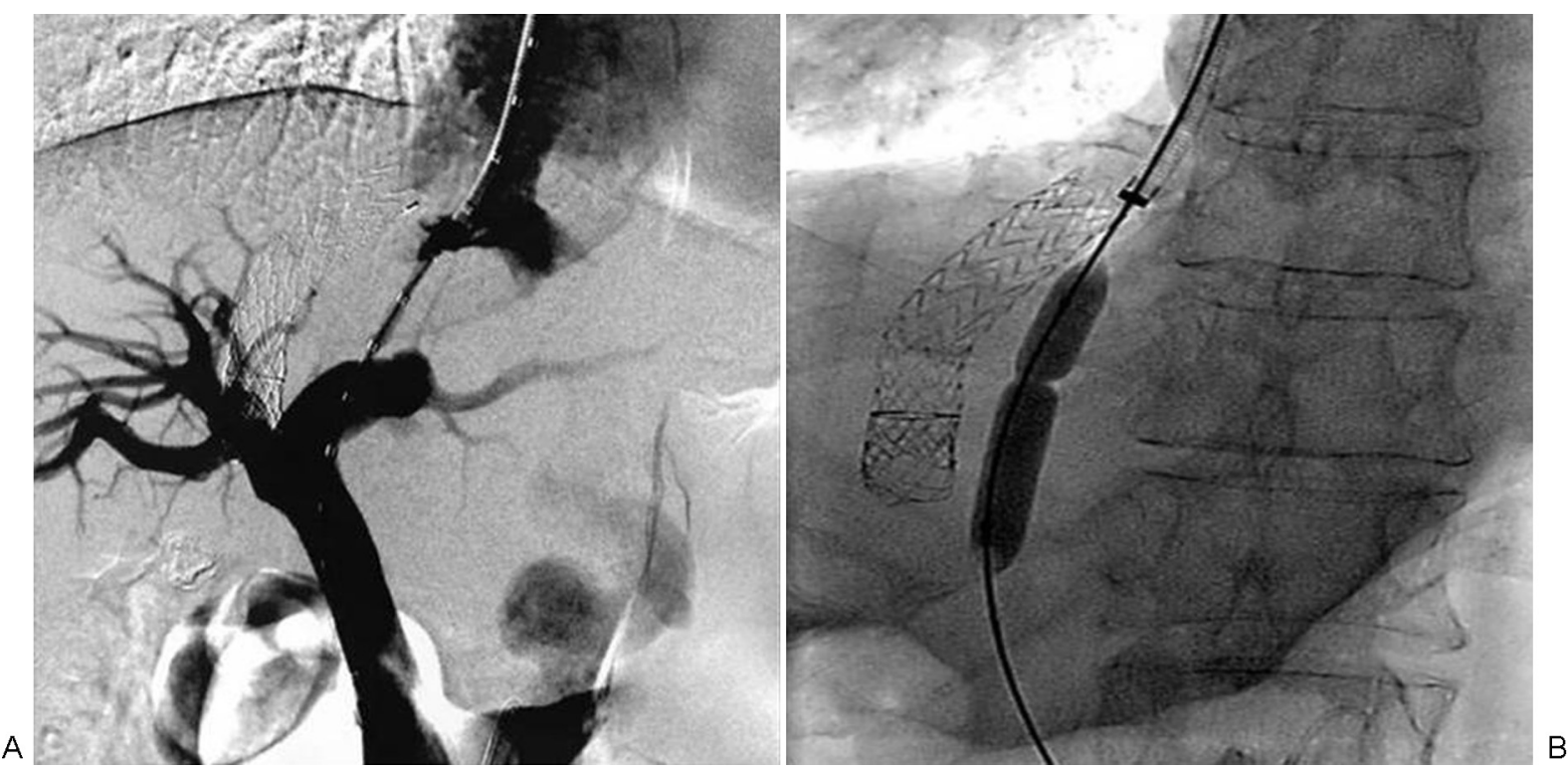

Fig. 1 (A) Parallel shunt created between the middle hepatic vein and the left portal vein. (B) Liver parenchymal tract dilated with a balloon. Note the waist at the left portal entry site. Note the previous occluded transjugular intrahepatic portosystemic shunt between the right hepatic vein and the right portal vein.

TIPS. The occluded TIPS was created between the right hepatic vein and the right portal vein. We planned a parallel TIPS between the middle hepatic vein and the left portal vein. The middle hepatic vein was cannulated and the Colapinto needle (Cook Medical, Bloomington, Illinois, United States) was directed anteriorly to puncture the left portal vein (-Fig. 1A) using the Haskal Liver Access Set (Cook Medical). A portal venography demonstrated enlarged esophageal varices (-Fig. 2). The parenchymal tract was dilated (-Fig. 1B) and a $10-\mathrm{mm}$ shunt was created by placing a TIPS Niti device (Taewoong Medical, Gyeonggi-do, South

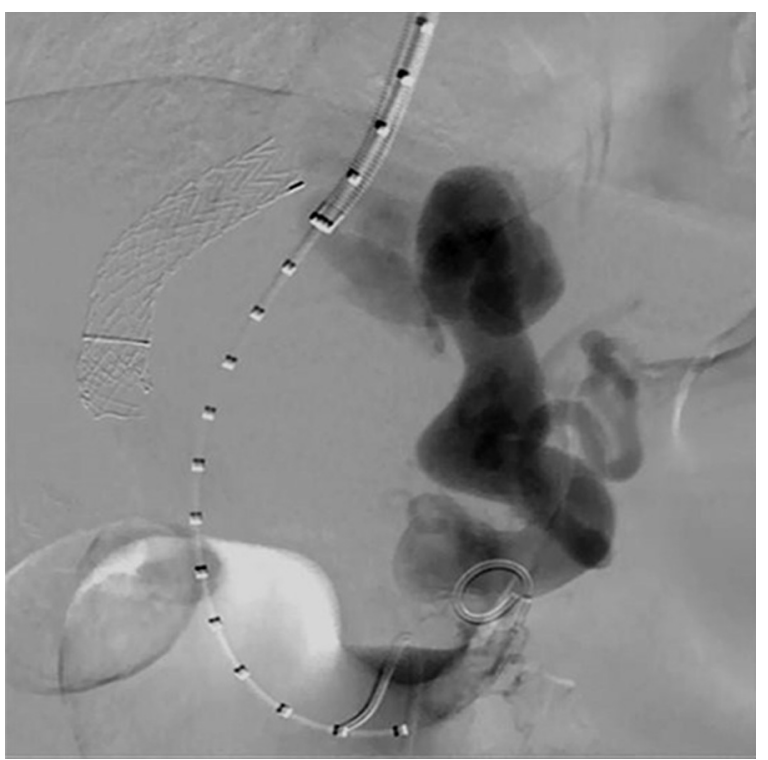

Fig. 2 Portal venography shows an enlarged left gastric vein and esophageal varices.
Korea) ( - Fig. 3A, B). The portal pressure decreased from $36 \mathrm{~mm} \mathrm{Hg}$ prior to TIPS creation to $8 \mathrm{~mm} \mathrm{Hg}$ post-TIPS. There was no antegrade flow in the esophageal varices post-TIPS and no embolization of the varices was performed. The patient had an uneventful recovery with no new episodes of gastrointestinal bleeding and resolution of ascites. The patient developed grade 1 encephalopathy, which was managed with medical therapy.

\section{Discussion}

TIPS is now considered an effective therapy in managing complications of portal hypertension. ${ }^{5-9}$ It is recommended that patients with TIPS are followed regularly by periodic duplex examination of the shunt. Secondary interventions may be required to maintain patency of the shunt. TIPS dysfunction can be secondary to acute stent thrombosis, hepatic venous outflow neointimal hyperplasia, and pseudointimal hyperplasia within the stentgraft. ${ }^{4}$ TIPS stent extending up to the hepatic vein ostium is associated with better long-term patency of TIPS than that terminating within the hepatic vein. ${ }^{9}$

An occluded or dysfunctional TIPS is treated based on the etiology of the occlusion. It is usually accessed through a transjugular venous access. Acute thrombosis is treated with pharmacological or mechanical thrombolysis followed by balloon angioplasty or stent extension, depending on the underlying pathology that resulted in acute thrombosis. Chronic stenosis or occlusion is treated with balloon angioplasty; however, many require repeat stenting. A percutaneous transhepatic or transplenic access to treat an occluded TIPS is reported and is applied when the transjugular route is unsuccessful. These alternative accesses are associated with high risk of bleeding especially in the presence of thrombocytopenia. If the existent shunt could not be recanalized, then 

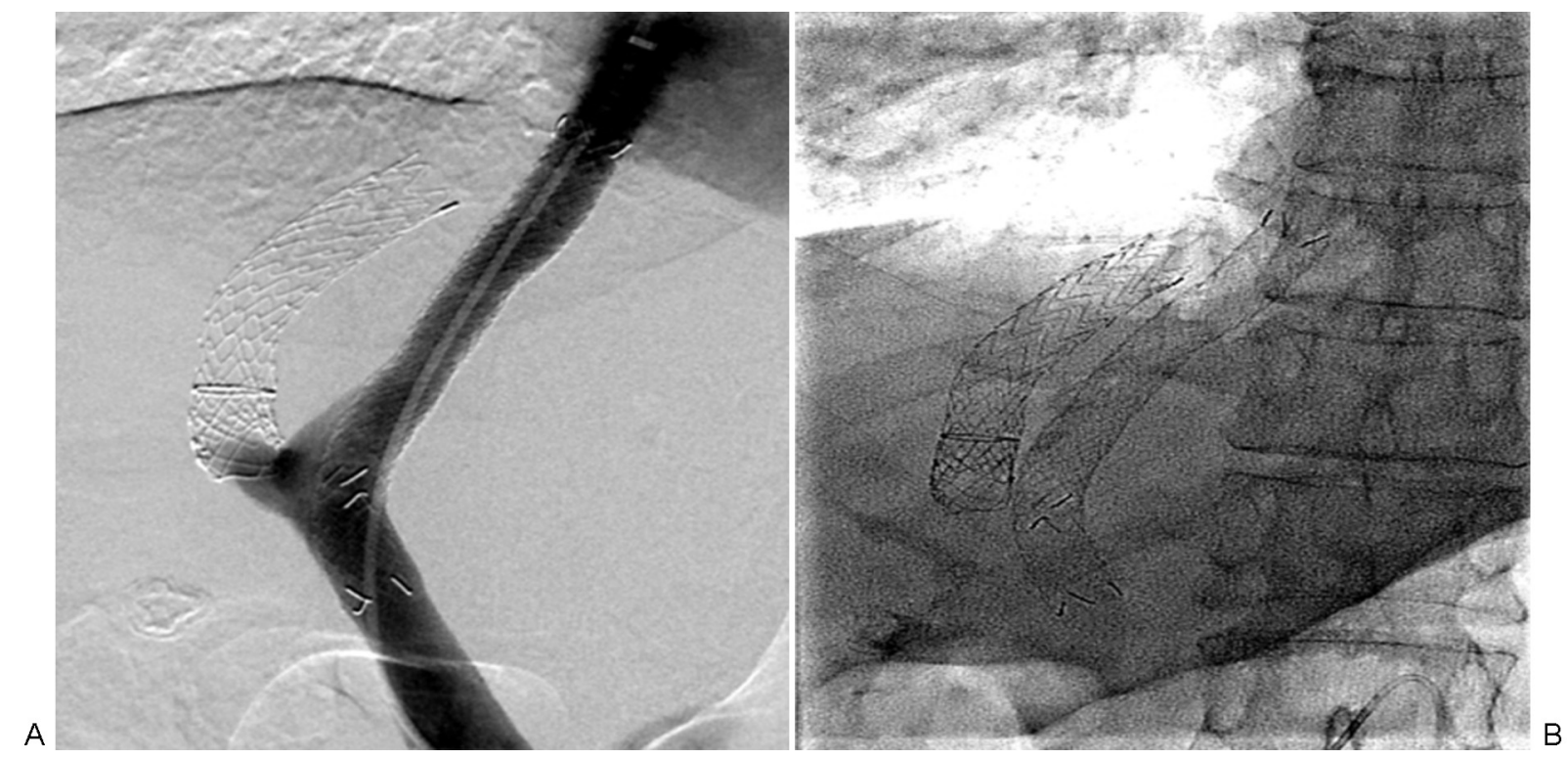

Fig. 3 (A) Posttransjugular intrahepatic portosystemic shunt (TIPS) venography shows a widely patent shunt with complete reversal of flow in the esophageal varices. (B) Parallel TIPS seen on fluoroscopy image.

a PS may be created to reduce portal pressure. In a series of 29 patients with PS placement, Dabos et $\mathrm{al}^{10}$ described that the PS patency was slightly superior to the patency of the preexisting TIPS. In another series of 40 patients, Helmy et $\mathrm{al}^{11}$ described that the natural history of PSs as opposed to the non-PSs after a follow-up of 11.6 months was similar.

In conclusion, PS creation with covered stent grafts, though technically challenging, is a safe and efficacious method in the management of refractory variceal bleeding and unsalvageable, preexisting TIPS dysfunction.

\section{References}

1 Garcia-Tsao G, Bosch J. Management of varices and variceal hemorrhage in cirrhosis. N Engl J Med 2010;362(9):823-832

2 Nishimine K, Saxon RR, Kichikawa K, et al. Improved transjugular intrahepatic portosystemic shunt patency with PTFE-covered stent-grafts: experimental results in swine. Radiology 1995; 196(2):341-347

3 Tanihata H, Saxon RR, Kubota Y, et al. Transjugular intrahepatic portosystemic shunt with silicone-covered Wallstents: results in a swine model. Radiology 1997;205(1):181-184

4 Cura M, Cura A, Suri R, El-Merhi F, Lopera J, Kroma G. Causes of TIPS dysfunction. AJR Am J Roentgenol 2008;191(6):1751-1757
5 Rössle M, Gerbes AL. TIPS for the treatment of refractory ascites, hepatorenal syndrome and hepatic hydrothorax: a critical update. Gut 2010;59(7):988-1000

6 García-Pagán JC, Caca K, Bureau C, et al; Early TIPS (Transjugular Intrahepatic Portosystemic Shunt) Cooperative Study Group. Early use of TIPS in patients with cirrhosis and variceal bleeding. N Engl J Med 2010;362(25):2370-2379

7 Feldstein VA, Patel MD, LaBerge JM. Transjugular intrahepatic portosystemic shunts: accuracy of Doppler US in determination of patency and detection of stenoses. Radiology 1996;201(1):141-147

8 Tzeng WS, Wu RH, Lin CY, et al. Prediction of mortality after emergent transjugular intrahepatic portosystemic shunt placement: use of APACHE II, Child-Pugh and MELD scores in Asian patients with refractory variceal hemorrhage. Korean J Radiol 2009;10(5):481-489

9 Clark TW, Agarwal R, Haskal ZJ, Stavropoulos SW. The effect of initial shunt outflow position on patency of transjugular intrahepatic portosystemic shunts. J Vasc Interv Radiol 2004;15(2 Pt 1): 147-152

10 Dabos KJ, Stanley AJ, Redhead DN, Jalan R, Hayes PC. Efficacy of balloon angioplasty, restenting, and parallel shunt insertion for shunt insufficiency after transjugular intrahepatic portosystemic stentshunt (TIPSS). Minim Invasive Ther Allied Technol 1998;7:287-293

11 Helmy A, Redhead DN, Stanley AJ, Hayes PC. The natural history of parallel transjugular intrahepatic portosystemic stent shunts using uncovered stent: the role of host-related factors. Liver Int 2006;26(5):572-578 